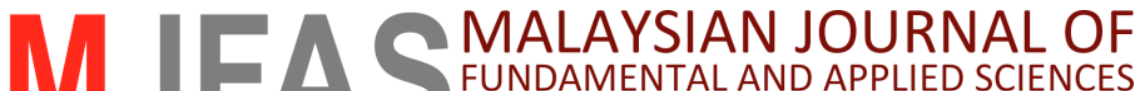

\section{MHD free convection flow of Casson fluid over a permeable nonlinearly stretching sheet with chemical reaction}

\author{
Imran Ullah a, Sharidan Shafie ${ }^{\mathrm{b},{ }^{*},}$, llyas Khan ${ }^{\mathrm{c}}$ \\ ${ }^{a, b}$ Department of Mathematical Sciences, Faculty of Science, Universiti Teknologi Malaysia, 81310 UTM Johor Bahru, Johor, Malaysia \\ c College of Engineering, Majmaah Univeristy, Majmaah, Saudi Arabia \\ * Corresponding author: sharidan@utm.my
}

\section{Article history}

Received 17 February 2017

Accepted 4 July 2017

\begin{abstract}
The problem of heat and mass transfer free convection flow of Casson fluid over a porous nonlinear stretching sheet in the presence of chemical reaction is investigated. Moreover the effect of magnetic field is also considered. The governing partial differential equations are transformed into ordinary differential equations by making use of suitable transformations and then solved numerically via Kellerbox method. The results for skin friction are compared with previous results of the existing literature. The results are also reflected in good agreement. It is noted that concentration of Casson fluid reduces rapidly by increasing Schmidt number and chemical parameter. Also, thermal Grashof number and mass Grashof number enhance the momentum boundary layer thickness, whereas increment in chemical reaction parameter reduces the heat transfer rate. Moreover, both the fluid velocity and wall shear stress are observed to be decreased with increment in suction/blowing parameter.
\end{abstract}

Keywords: Casson fluid, MHD, suction/blowing, free convection

\section{INTRODUCTION}

The study of non-Newtonian fluids have gained attraction due to its wide range application in various industries, such as design of solid matrix heat, nuclear waste disposal, chemical catalytic reactors, geothermal energy production, ground water hydrology, transpiration cooling, petroleum reservoirs, etc. These fluids are more complicated as compared to Newtonian fluids because of highly non-linear governing equations. Several models have been put forward in the study of non-Newtonian fluids, but yet not a single model is developed that exhibits all properties of non-Newtonian fluids. In literature, the simplest model is the Maxwell model. Among numerous nonNewtonian fluids, there is another fluid known as Casson fluid. Casson fluid is a shear thinning fluid and is defined as it has an infinite viscosity at zero rate of shear, a yield stress below which no flow occurs and a zero viscosity at an infinite rate of shear. The Casson model, also called rheological model was originally developed by Casson (Casson, 1959) for viscous suspension of cylindrical particles (Reher et al., 1969). Common examples of Casson fluid are honey, jelly, soup, tomato sauce, concentrated fruit juices, etc. Also, it is the most appropriate rheological model for blood and chocolate. Furthermore, Casson fluid possesses yield stress and has great importance in polymer processing industries and biomechanics. Based on its important industrial applications, several researchers (Singh, 2011; Gaur and Gupta, 2014; Hussanan et al., 2016; Raju and Sandeep, 2017) have included Casson fluid in their work in the recent years.

Boundary layer flow over a stretching surface has several engineering and industrial applications, for example, in cooling bath, Aerodynamic extrusion, plastic sheet, metallurgical process, glass blowing, manufacturing of rubber and plastic sheets, crystal growing, the sheets are continuously stretched. During manufacturing, sheets are stretched continuously in order to achieve the desired thickness. It shows that final product depends upon the stretching and cooling rate of the sheet. In the pioneer work of (Sakiadis, 1961), he analyzed numerically the two dimensional flow over continuous stretched surface with the help of similarity transformations. Later on, (Gupta and Gupta, 1977) extended this problem to the flow subject to suction/injection, and discovered that the stretching sheet velocity needs not be linear, it can be quadratic, exponential and non-linear. Keeping this in mind, (Kumaran and Ramanaiah, 1996) and (Kechil and Hashim, 2009) studied viscous flow over quadratic stretching sheet. (Magyari and Keller, 1999), (Sajid and Hayat, 2008) and (Pramanik, 2014) studied heat and mass transfer characteristics in boundary layer flow over exponentially stretching sheet. (Vajravelu, 2001), (Cortell, 2007), (Hsiao, 2010), (Prasad et al., 2010) and (Jat et al., 2014) investigated heat transfer effects in two dimensional incompressible flow of viscous fluid over non-linearly stretching sheet. On the other hand, (Mukhopadhyay, 2013) studied numerically two dimensional boundary layer flow of Casson fluid over non-linearly stretching sheet.

The impact of magnetohydrodynamic (MHD) and chemical reaction with combined heat and mass transfer has immense importance in many areas of engineering and industries. This phenomenon plays an important role in chemical industry, cooling of nuclear reactors, MHD power generation, MHD pumps, packed-bed catalytic reactor, formation and dispersion of fog, high speed plasma, cosmic jets, enhanced oil recovery, distribution of temperature and moisture over agriculture fields, cooling of nuclear reactors, manufacturing of ceramics, underground energy transport, food processing and cooling towers, etc. In the recent years, several researchers have considered combined effect of magnetic field and chemical reaction on boundary layer flows. (Chamkha et al., 2004) carried out an analysis for the chemical reaction effect on boundary layer flow over a stretching sheet under the influence of magnetic field. They found that magnetic field enhances the skin friction significantly. (Raptis and Perdikis, 2006) studied the chemical reaction effect on laminar boundary layer flow over non-linearly stretching sheet under the influence of magnetic fluid. 
The combined effect of magnetic and chemical reaction on boundary layer flow of Casson fluid due to a linear stretching sheet has been discussed by (Shehzad et al., 2013). They found that suction parameter reduces concentration of the fluid. Recently, (Mangathai et al., 2015) investigated numerically the effect of chemical reaction on boundary layer flow caused by a linear stretching sheet embedded in a porous medium under the influence of magnetic field. They conclude that the presence of magnetic field and chemical reaction reduce the velocity across the boundary.

On the other hand, heat transfer in free convection flows often involves large temperature differences. Its practical applications can be found in various branches of industry, such as the metallurgical, chemical, mechanical, and food industries. Free convection or natural convection occurs only due to the presence of buoyancy forces. It is worth mentioning here that the rate of heat transfer by free convection is generally smaller than that of forced convection. Furthermore, the passive nature of free convection effectively increases system reliability. Natural circulation in flow loops occurs due to free convection, which plays a vital role in nuclear plants during shutdown. Several studies have been declared in the last few years on free convection flow. (Makinde, 2005) discussed the combined heat transfer effect on free convectional flow due to vertical porous plate in the presence of magnetic field and thermal radiation and concluded that Prandtl number has no significant effect on Sherwood number. (Mahdy, 2014) investigated three dimensional natural convection flow of Casson fluid past vertical stretching sheet with heat transfer. He noticed that velocities at the surface are higher in case of suction, whereas linear in case of injection.

To the best of authors' knowledge, free convection flow of Casson fluid over porous nonlinearly stretching sheet in the presence of chemical reaction has not been perceived previously. The purpose of present work is to investigate the combined heat and mass transfer on free convectiona flow of Casson fluid over non-linearly stretching sheet under the influence of chemical reaction and magnetic field. The system of mass conservation, momentum heat and concentration equations are transformed to ordinary differential equation using suitable similarity transformations. The resulting transformed equations are then solved numerically by Keller box method (Cebeci and Bradshaw, 1988).

\section{MATHEMATICAL FORMULATION}

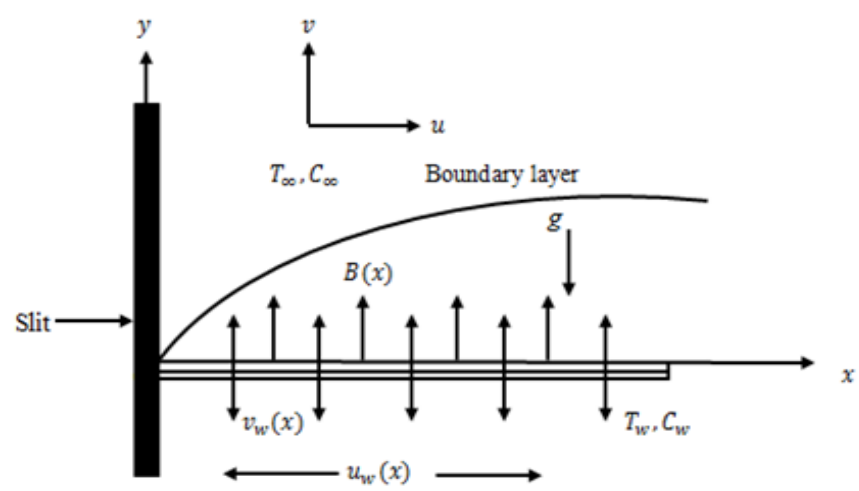

Fig. 1 Sketch of physical problem and coordinate system.

Consider the steady two dimensional and incompressible free convectional flow of Casson fluid over non-linearly stretching sheet under the influence of magnetic field and chemical reaction. It is assumed that the sheet is stretched in the $x$ - direction and $y$-direction is taken for fluid flow with the origin fixed (see Fig. 1). The stretching sheet and wall mass suction velocities are assumed to be of the form $u_{w}(x)=c x^{n}$ and $v_{w}(x)=V_{0} x^{\frac{n-1}{2}}$ respectively, where $c$ and $V_{0}$ are constants and $n(n \geq 0)$ is the non-linear stretching sheet parameter. A non-uniform transverse magnetic field of strength $B_{0}$ is applied normal to the sheet. The induced magnetic field is neglected due to small magnetic Reynolds number. The surface of stretching sheet is heated by the convection of hot fluid temperature $T_{f}=T_{\infty}+A x^{2 n-1}$. The temperature and the species concentration maintained at a boundary and free stream are $T_{\infty}, C_{w}$ and $C_{\infty}$, respectively.

The governing equations of continuity, momentum heat and mass transfer in usual notation can be written as:

$$
\frac{\partial u}{\partial x}+\frac{\partial v}{\partial y}=0
$$

$$
\begin{gathered}
u \frac{\partial u}{\partial x}+v \frac{\partial u}{\partial y}=v\left(1+\frac{1}{\beta}\right) \frac{\partial^{2} u}{\partial y^{2}}-\frac{\sigma B^{2}(x)}{\rho} u+g \beta_{T}\left(T-T_{\infty}\right) \\
+g \beta_{C}\left(C-C_{\infty}\right),
\end{gathered}
$$

$u \frac{\partial T}{\partial x}+v \frac{\partial T}{\partial y}=\alpha \frac{\partial^{2} T}{\partial y^{2}}$

$u \frac{\partial C}{\partial x}+v \frac{\partial C}{\partial y}=D \frac{\partial^{2} C}{\partial y^{2}}-R\left(C-C_{\infty}\right)$

In the above expressions $u$ and $v$ denote the velocity component in $x$ - and $y$-directions respectively, $\rho$ is the fluid density, $v$ is kinematic viscosity, $\beta$ is the Casson parameter, $\sigma$ is the electrically conductivity of the fluid, $B(x)=B_{0} x^{\frac{n-1}{2}}$ is the magnetic field with constant magnetic strength $B_{0}, g$ is the gravitational force due to acceleration, $\beta_{T}$ is the volumetric coefficient of thermal expansion, $\beta_{C}$ is the volumetric coefficient of expansion with concentration, $T$ is the fluid temperature, $\alpha=\frac{k}{\rho c_{p}}$ is the thermal diffusivity of the Casson fluid, $k$ is the thermal conductivity of fluid, $c_{p}$ is the specific heat at constant pressure, $C$ is concentration of the fluid, $D$ is the diffusion coefficient of species in the fluid and $R$ is the solute reaction rate. The corresponding boundary conditions for the problem can be written as follows:

$$
\begin{aligned}
& \left.u(x)=c x^{n}, v=-v_{w}(x), k \frac{\partial T}{\partial y}=-h_{f}\left(T_{f}-T\right)\right\}, \\
& C=C_{w}=C_{\infty}+B x^{2 n-1} \text { at } y=0 \\
& u \rightarrow 0, T \rightarrow T_{\infty}, \mathrm{C} \rightarrow C_{\infty} \text { as } y \rightarrow \infty
\end{aligned}
$$

Here $v_{w}(x)>0$ is the suction velocity and $v_{w}(x)<0$ is the blowing velocity.

We introduce the following dimensionless variables,

$$
\begin{aligned}
& \psi=\sqrt{\frac{2 v c x^{n+1}}{n+1}} f(\eta), \eta=\sqrt{\frac{(n+1) c x^{n-1}}{2 v}} y, \theta=\frac{T-T_{\infty}}{T_{f}-T_{\infty}}, \\
& \phi=\frac{C-C_{\infty}}{C_{w}-C_{\infty}}
\end{aligned}
$$

where the stream function $\psi$ satisfies Eq. (1) and given as:

$$
u=\frac{\partial \psi}{\partial y}, v=-\frac{\partial \psi}{\partial x}
$$

From Eqs. $(2-7)$, the system of equations along with the boundary conditions can be written as: 


$$
\begin{aligned}
& \left(1+\frac{1}{\beta}\right) f^{\prime \prime \prime}+f f^{\prime \prime}-\frac{2 n}{n+1} f^{\prime 2}-M f^{\prime}+G r \theta+G m \phi=0 \\
& \theta^{\prime \prime}+\operatorname{Pr} f \theta^{\prime}-\frac{2(2 n-1)}{n+1} \operatorname{Pr} f^{\prime} \theta=0 \\
& \phi^{\prime \prime}+S c f \phi^{\prime}-\frac{2(2 n-1)}{n+1} S c f^{\prime} \phi-\gamma S c \phi=0 \\
& f(\eta)=S, f^{\prime}(\eta)=1, \theta^{\prime}(0)=-B i[1-\theta(0)], \phi(\eta)=1 \text { at } \eta=0 \\
& f^{\prime}(\eta)=0, \theta(\eta)=0, \phi(\eta)=0 \text { as } \eta \rightarrow \infty
\end{aligned}
$$

where primes indicate derivatives with respect to $\eta$ and the parameters are defined as:

magnetic parameter, $M=\frac{2 \sigma B_{0}^{2}}{\rho c(n+1)}$, thermal Grashof number, $G r=\frac{2 g \beta_{T} A}{c^{2}(n+1)}$, mass Grashof number, $G m=\frac{2 g \beta_{C} B}{c^{2}(n+1)}$, Prandtl number, $\operatorname{Pr}=\frac{v}{\alpha}$, Schmidt number, $S c=\frac{v}{D}$, chemical reaction parameter, $\quad \gamma=\frac{2 v R}{(n+1) u_{w}{ }^{2}(x)}, \quad$ suction/blowing parameter, $S=-\frac{\sqrt{2} V_{0}}{\sqrt{c v(n+1)}}$ such that $S>0$ indicates suction, $S=0$ represents impermeable wall and $S<0$ indicated blowing at the surface and Biot number, $B i=\frac{h_{0}}{k}\left[\frac{2 v}{c(n+1)}\right]^{1 / 2}$

The parameters with physical interests are the skin friction coefficient, local Nusselt number and Sherwood number, and are given as:

$$
C f_{x}=\frac{\tau_{w}}{\rho u_{w}^{2}}, \quad N u_{x}=\frac{x q_{w}}{\alpha\left(T_{f}-T_{\infty}\right)}, S h_{x}=\frac{x q_{s}}{D\left(C_{w}-C_{\infty}\right)}
$$

where $\tau_{w}, q_{w}$ and $q_{s}$ are the wall skin friction, heat flux and mass flux, respectively, defined by:

$$
\tau_{w}=\mu_{B}\left(1+\frac{1}{\beta}\right)\left[\frac{\partial u}{\partial y}\right]_{y=0}, q_{w}=-\alpha\left(\frac{\partial T}{\partial y}\right)_{y=0}, q_{s}=-D\left(\frac{\partial C}{\partial y}\right)_{y=0}
$$

Using Eq. (7) into Eq. (15), we get the following non dimensional skin friction coefficient, local Nusselt number and local Sherwood number:

$$
\begin{aligned}
& (\operatorname{Re})^{1 / 2} C f_{x} \sqrt{\frac{2}{n+1}}=\left(1+\frac{1}{\beta}\right) f^{\prime \prime}(0),(\operatorname{Re})^{-1 / 2} N u_{x} \sqrt{\frac{2}{n+1}}=-\theta^{\prime}(0), \\
& (\operatorname{Re})^{-1 / 2} S h_{x} \sqrt{\frac{2}{n+1}}=-\phi^{\prime}(0)
\end{aligned}
$$

\section{NUMERICAL SCHEME}

The numerical solutions for the system of Eqs. (9)-(13) are obtained using Keller-box method. The detail work of this method can be visualized in the book of Cebeci and Bradshaw (1988). The advantage of using this method is that, it is unconditionally stable and more appropriate to apply to parabolic equations. Keller-box method comprise of following four steps to obatain numerical solutions: (i) At first, the governing equations are written interms of first order equations.

(ii) Center difference scheme about the mid point is used to approximate the first-order system.

(iii) Next, Newton's method is used to linearize the system of equations and express them in matrix-vector form.

(iv) Finally, the linear system can be used via block tri-diagonal techneque.

\section{RESULTS AND DISCUSSION}

In order to insight the behavior of velocity, temperature and species concentration profiles of the physical problem, numerical calculations are carried out for various values of Casson fluid parameter $\beta$, magnetic parameter $M$, nonlinear stretching parameter $n$, thermal Grashof number $G r$, mass Grashof number $G m$, Prandtl number Pr, Schmidt number $S c$, chemical reaction parameter $\gamma$ and suction/blowing parameter $S$. Results for skin friction coefficient, local Nusselt number and local Sherwood number are computed and compared with the previously reported results, and are displayed in Table 1.

Table 1 Comparison of $-f^{\prime \prime}(0)$ for different values of $n$ with $M=S=G r=G m=\gamma=0$ and $\beta \rightarrow \infty$.

\begin{tabular}{ccc}
\hline \multicolumn{3}{c}{$-f^{\prime \prime}(0)$} \\
\hline$n$ & Cortell (2007) & Present results \\
\hline 0.0 & 0.627547 & 0.6276 \\
0.2 & 0.766758 & 0.7668 \\
0.5 & 0.889477 & 0.8895 \\
1 & 1.0 & 1.0 \\
3 & 1.148588 & 1.1486 \\
10 & 1.234875 & 1.2349 \\
100 & 1.276768 & 1.2768 \\
\hline
\end{tabular}

Table 1 presents the values of skin friction coefficient and local Nusselt number for different values of $n$ and $\operatorname{Pr}$, respectively. The present results are compared with the previously reported results of (Cortell, 2007). It is noticed that the results are in excellent agreement. Figures (2-5) are plotted to study dimensionless velocity profile for various effects of $\beta, M, G r$ and $G m$. Fig. 2 demonstrates the variation of velocity profile for several values of $\beta$ for $n=1$ and . It is worth mentioning here that $\beta \rightarrow \infty$ represent Newtonian flow. It is observed that fluid velocity declines with the increase of $\beta$ reason behind this phenomenon is that increasing values of $\beta$ lead to enhancing the plastic dynamic viscosity of fluid that produces a drag force in the fluid and as a consequence velocity of fluid falls. It is also observed from this figure that increasing values of $n$ decreases the velocity in the boundary layer. Since friction at wall $(\eta=0)$ rises with increase in $n$, which leads to increase in velocity boundary layer thickness.

The velocity is found to be reduced with increasing values of (see Fig. 3) as expected. It is due to the reason that drag force named Lorentz force is produced by applying magnetic field normal to electrically conducting fluid in the boundary layer, in result slower down the velocity field. It is also determined from the graph that velocity of fluid reduces in case of suction while enhances in case of blowing. Physically, stronger blowing pushes the heated fluid away from the wall where flow is accelerated due to low viscosity. Furthermore, the boundary layer thickness decreases in case of wall suction $(S>0)$. 


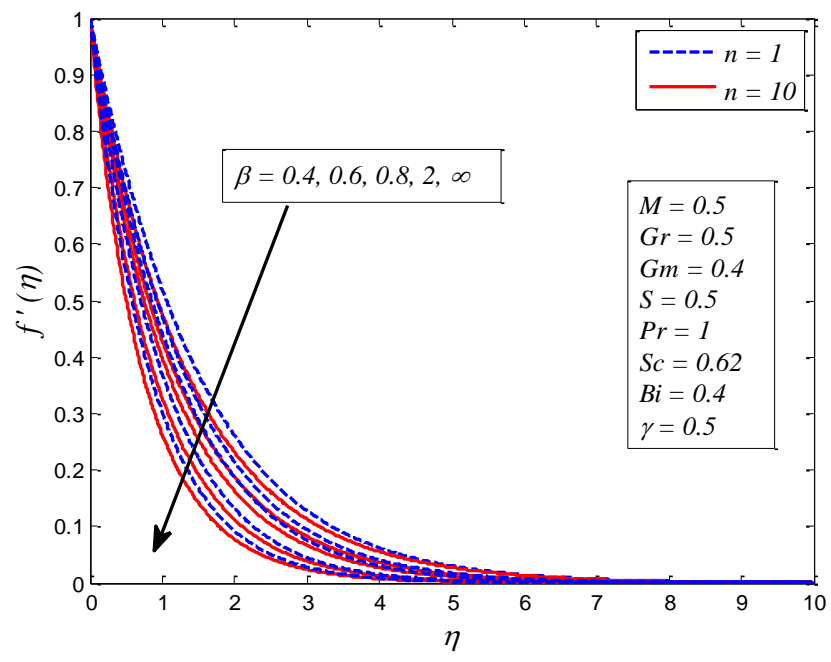

Fig. 2 Effect of $\beta$ on velocity for different $n$.

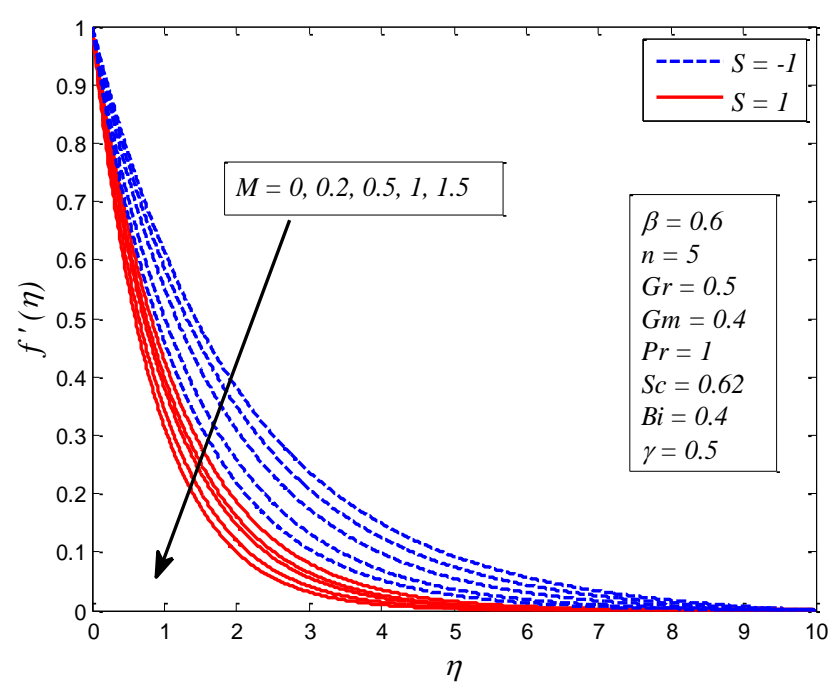

Fig. 3 Effect of $M$ on velocity for different $S$.

The effect of $G r$ on velocity profile is plotted in Fig. 4. It is observed that the velocity increases with the increase of $\mathrm{Gr}$ in case of suction as well as blowing. Also, a rapid increase in velocity for the case of blowing is seen. The similar pattern of velocity profile is observed for $G m$ (see Fig. 5). This is due to the fact that momentum boundary layer thickness increases with increasing values of $\mathrm{Gr}$ and Gm .

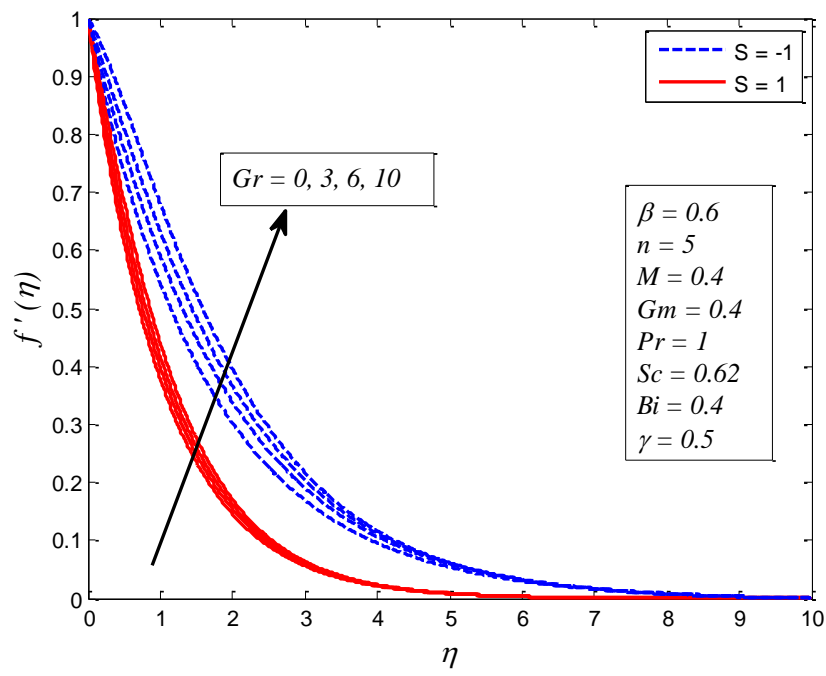

Fig. 4 Effect of $G r$ on velocity for different $S$.

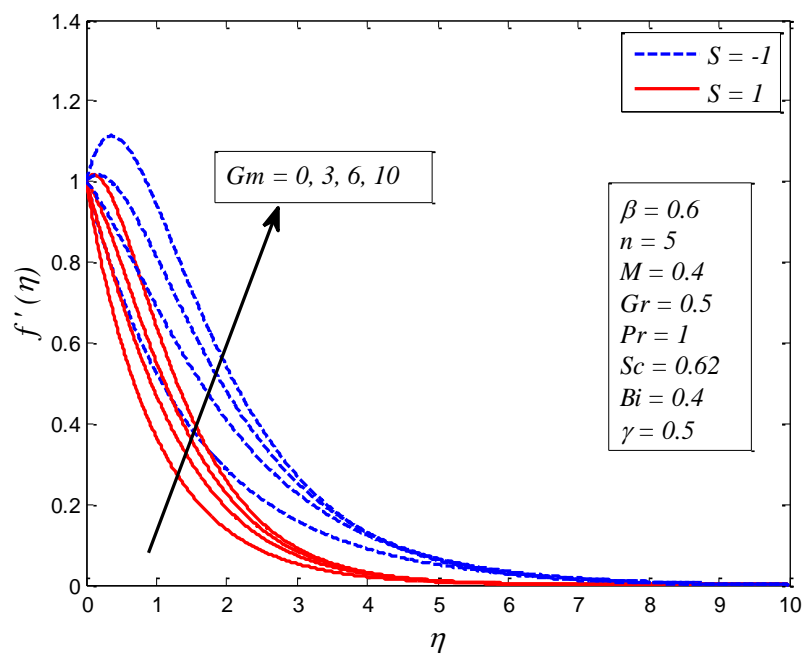

Fig. 5 Effect of $G m$ on velocity for different $S$.

Figures (6-8) are depicted to analyze various effects of $\beta$, Pr and $B i$ on temperature profile. From Fig. 6 , it is noted that the dimensionless temperature increases with the increase of $\beta$ in case of linear and nonlinear stretching sheet. The existence of yield stress reduces rate of heat transfer and consequently fluid temperature rises.

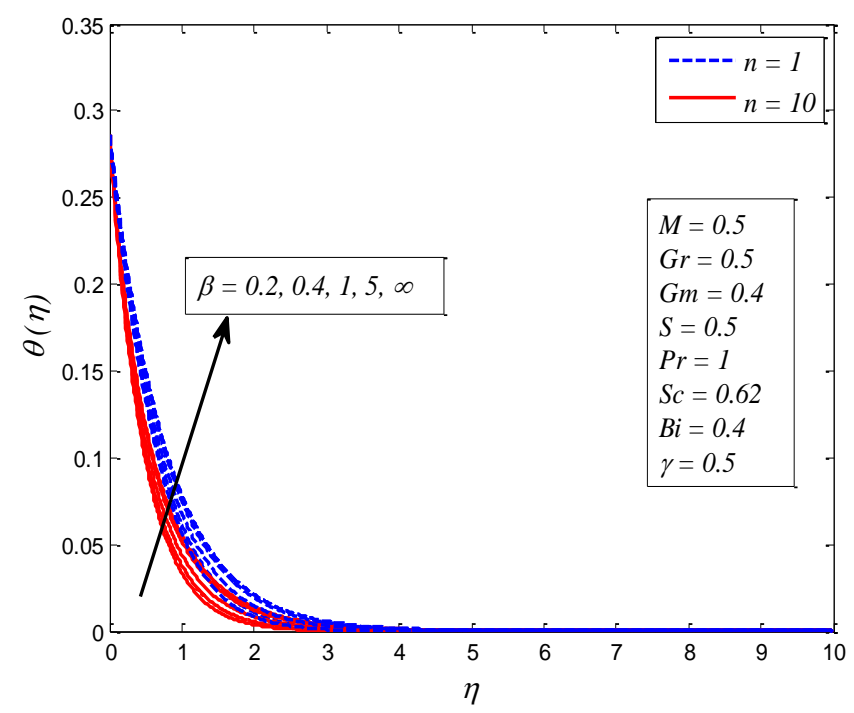

Fig. 6 Effect of $\beta$ on velocity for different $n$.

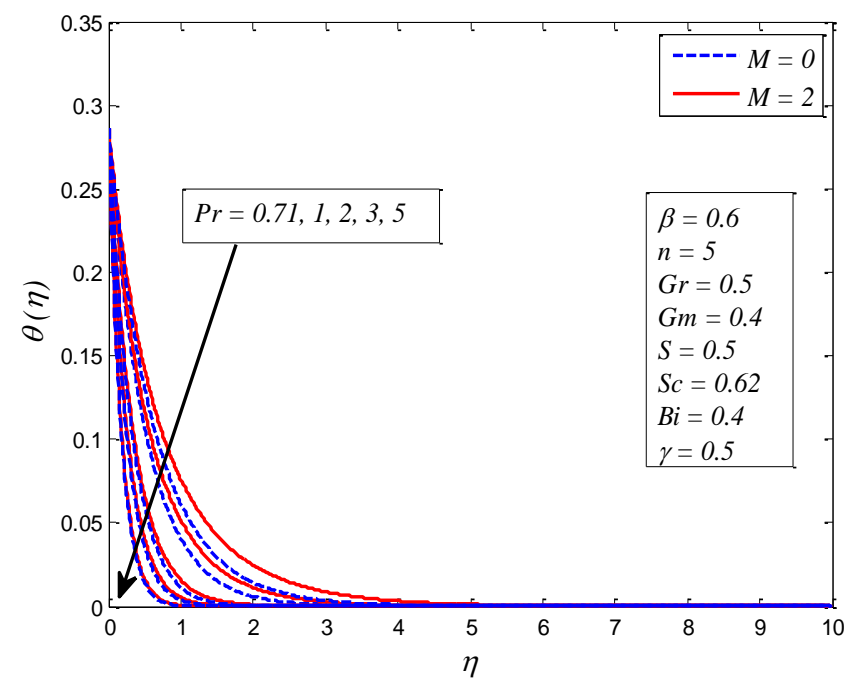

Fig. 7 Effect of $\operatorname{Pr}$ on velocity for different $M$. 


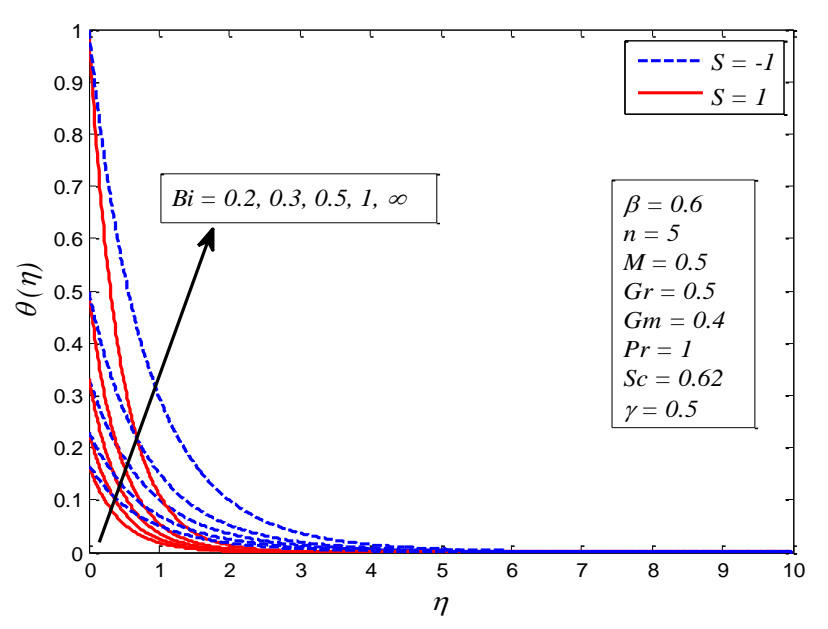

Fig. 8 Effect of $B i$ on velocity for different $S$.

As expected, the dimensionless temperature decreases with the increase of $\operatorname{Pr}$ for $M=0$ and $M \neq 0$ (see Fig. 7). Also, boundary layer thickness decreases and getting closer and closer to wall more rapidly for large values of Prandtl number. As Prandtl number is defined as the ratio between momentum diffusion to thermal diffusion.

Thus, thermal diffusion decreases as Prandtl number increases. Physically, large Prandtl number in the fluid flow transfer heat faster to the surface of the fluid. Since lower suction tends to larger thermal boundary layer thickness. This implies that thickness of thermal boundary layer in case of suction is thinner than that of blowing. In other words, thickness of thermal boundary layer decreases in case of suction when the surrounding fluid brought closer to stretching sheet surface at ambient condition. It is also noticed from this figure that temperature is higher for higher values of $M$.

The variation of $B i$ on dimensionless temperature for $S=-1$ and $S=1$ is displayed in Fig. 8. It is found that temperature is higher for larger values of $B i$. It is an agreement with the fact that heat transfer coefficient increases as Biot number increased, and as a consequence temperature rises in the flow field. This graph also clears that the dimensionless temperature falls in the case of suction and rise in the case of blowing. Since lower suction tends to larger thermal boundary layer thickness. This implies that thickness of thermal boundary layer in case of suction is thinner than that of blowing. In other words, thickness of thermal boundary layer decreases in case of suction when the surrounding fluid brought closer to stretching sheet surface at ambient condition.

Figures (9-11) are plotted to analyze the effects of $\beta, S c$ and $\gamma$ on concentration profile, respectively. Fig. 9 illustrates the influence of $\beta$ on concentration profile for the case linear and nonlinear stretching sheet. In both cases, concentration of the fluid increases with the increase in $\beta$. Also, the present phenomenon reduces to Newtonian fluid when $\beta \rightarrow \infty$. Furthermore, larger values of $\beta$ have no significant effect of concentration of the fluid across the boundary.

Fig. 10 indicates that concentration of the fluid and boundary layer thickness decreases with increase of $S c$ across the boundary. Also, boundary layers squeezing closer and closer to the wall for higher values of $S c$. As Schmidt number has inverse relation with mass diffusion coefficient, thus higher Schmidt number correspond to decrease in mass diffusion coefficient, which in result reduces concentration of the fluid. This figure also shows that increasing values of $M$ enhances the thermal boundary layer thickness.

To insight the effect of $\gamma$ on concentration profile, Fig. 11 is depicted. It is seen that increasing values of $\gamma$ declines the concentration of the fluid across the boundary for suction $(S=-1)$ as well as blowing ( $S=1)$. It is due to reason that interfacial mass rate increase as chemical reaction parameter increases. Physically, friction between fluid layers increases as $S$ increases, which show resistance to the fluid. Consequently, the fluid concentration decreases.

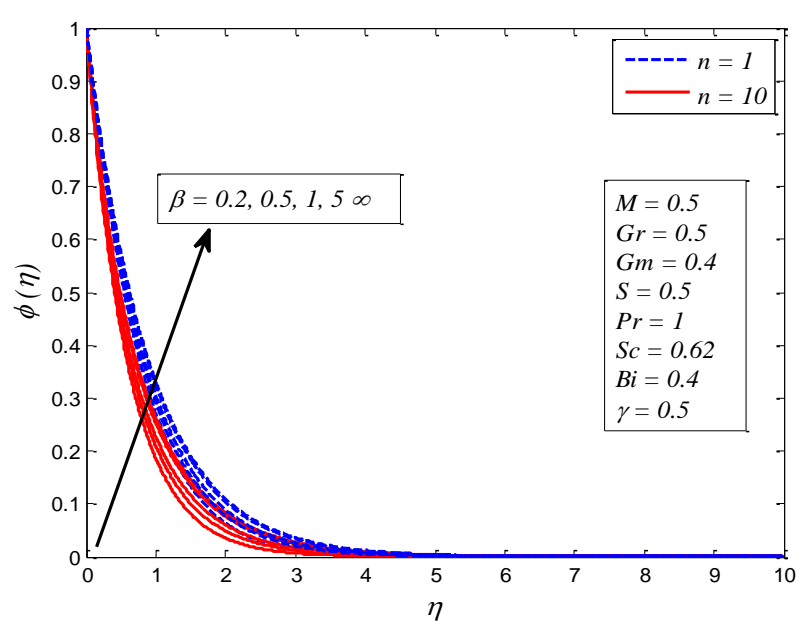

Fig. 9 Effect of $\beta$ on velocity for different $n$.

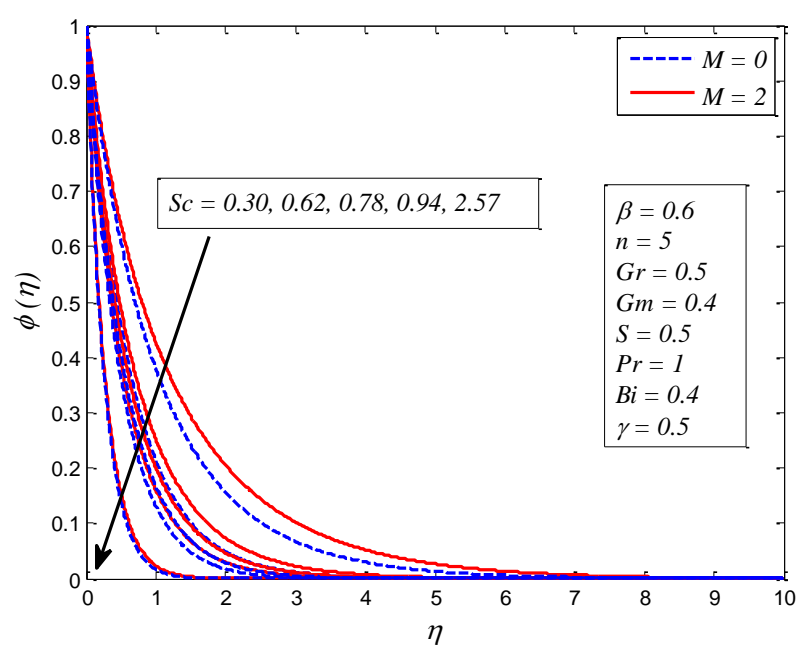

Fig. 10 Effect of $S c$ on velocity for different $M$.

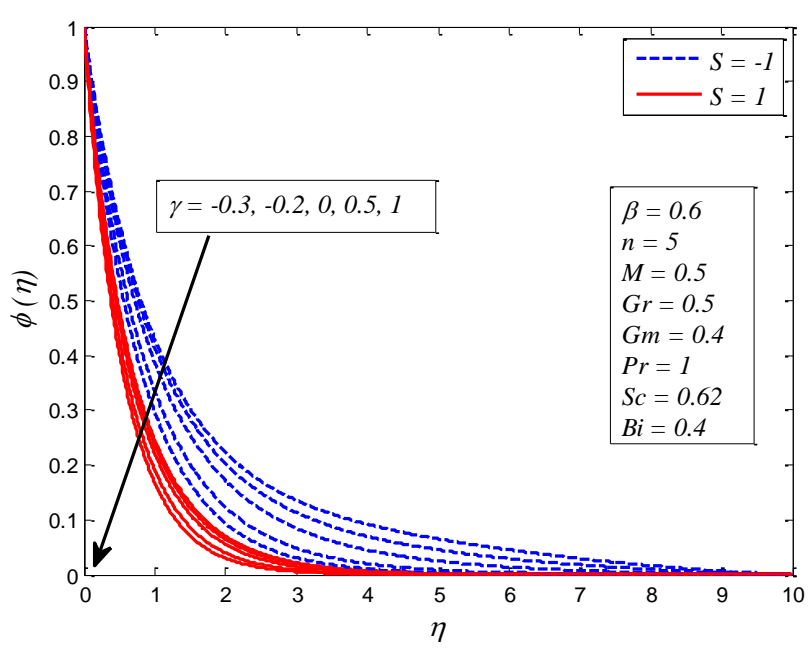

Fig. 11 Effect of $\gamma$ on velocity for different $S$.

Figures (12-14) are plotted to analyze the skin friction coefficient, local Nusselt number and local Sherwood number for various values of $\beta, M, S, \operatorname{Pr}$ and $\gamma$. Fig. 12 indicates the variation of local skin friction coefficients for various values of $S, M$ and $\beta$. It needs to be mentioned here that all values of skin friction coefficient are negatives which shows that the stretching sheet exerts a drag force on working fluid and opposite to this for positive values. It is seen that absolute friction factor enhances with the increase of $S, M$ and $\beta$. Fig. 13 exhibits that rate of heat transfer enhances with the increase of $\operatorname{Pr}$ and 
$S$ whereas decreases with the increase of $\beta$. Finally, from Fig. 14, it is observed that mass transfer rate is higher for increasing values of $\gamma$ and $S$ while reduces with the increase of $\beta$.

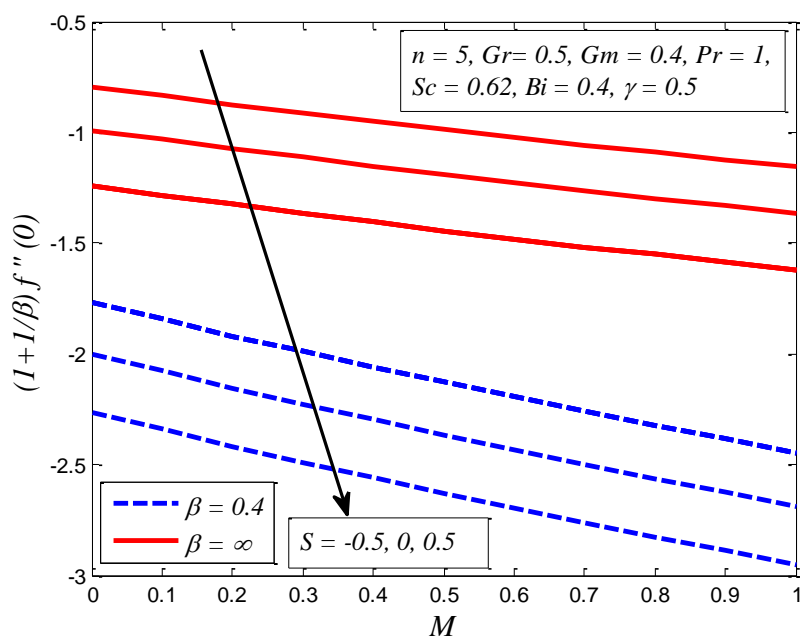

Fig. 12 Variation of skin friction coefficient for various values of $S$, $\beta$, and $M$.

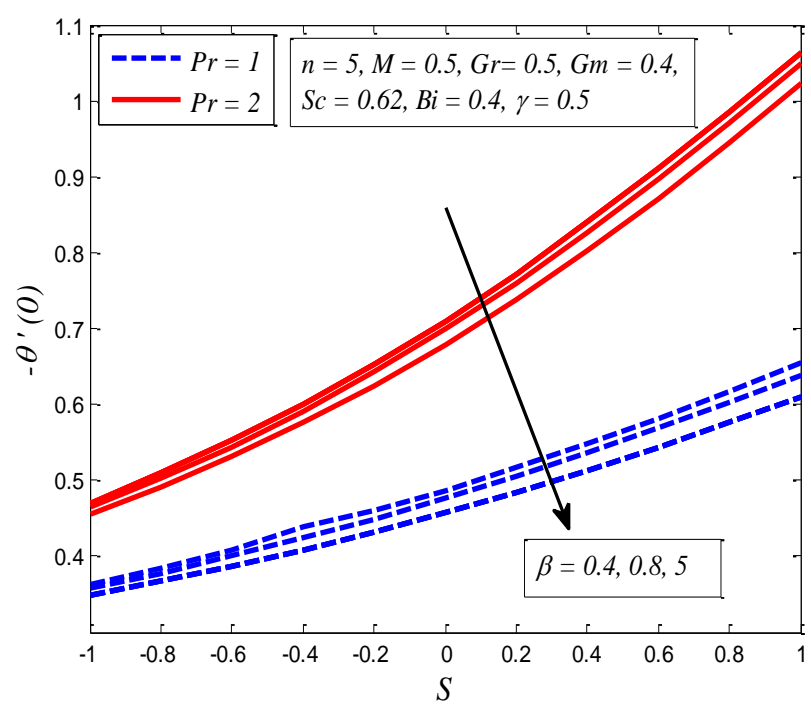

Fig. 13 Variation of Nusselt number for various values of $\operatorname{Pr}, \beta$ and $S$.

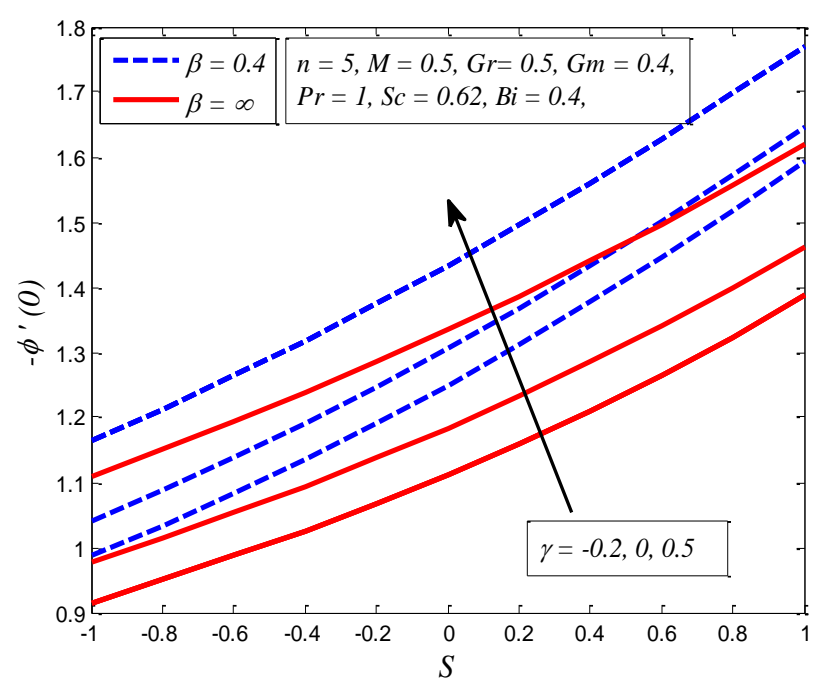

Fig. 13 Variation of Sherwood number for various values of $\gamma, \beta$ and $S$.

\section{CONCLUSION}

The present study investigates the numerical solution of free convection flow of Casson fluid over non-linearly stretching sheet in the presence of chemical reaction and transverse magnetic field. Physically, the effect of Casson parameter $\beta$, magnetic parameter $M$, nonlinear stretching parameter $n$, thermal Grashof number $G r$, mass Grashof number $G m$, Prandtl number Pr, Schmidt number $S c$, chemical reaction parameter $\gamma$ and suction/blowing parameter $S$ are studied in detail and the results are discussed graphically. The main findings of this study can be summarized as follows:

(i) The momentum boundary layer thickness decreases with the increase in $\beta$ and $S$.

(ii) Fluid velocity is found to be enhanced with $\mathrm{Gr}$ and $\mathrm{Gm}$.

(iii) The thermal boundary layer becomes thicker as $\beta, M$ and $S$ increased.

(iv) Concentration of the fluid is observed to be higher with increase in $\beta$ and $M$.

(v) Concentration of the fluid decreases more rapidly with the increase of $S c$ and $\gamma$ as compared to other parameters.

(vi) The wall shear stress is found to be more pronounced with an increase in $\beta$ as compared to heat and mass transfer rate.

\section{ACKNOWLEDGEMENT}

The authors would like to acknowledge Ministry of Higher Education (MOHE) and Research Management Centre Universiti Teknologi Malaysia (UTM) for the financial support through vote numbers 4F713 and $13 \mathrm{H} 74$ for this research..

\section{REFERENCES}

Casson, N., 1959. A flow equation for pigment-oil suspensions of the printing ink type. In: Mill, C. C., Ed., Rheology of Disperse Systems, Pergamon Press, Oxford, pp.84-104.

Cebeci, T. and Bradshaw, P., 1988. Physical and computational aspects of convective heat transfer 1 st ed., Springer-Verlag, New York.

Chamkha, A. J., Takhar, H. S. and Bég, O. A., 2004. Radiative free convective non-Newtonian fluid flow past a wedge embedded in a porous medium. international Journal of Fluid Mechanics Research, 31(2), pp.101-115. doi: 10.1615/InterJFluidMechRes.v31.i2.10.

Cortell, R., 2007. Viscous flow and heat transfer over a nonlinearly stretching sheet. Applied Mathematics and Computation, 184(2), pp.864-873.

Gaur, M. and Gupta, M.K., 2014. A Casson fluid model for the steady flow through a stenosed blood vessel 2 mathematical formulation. British Journal of Mathematics \& Computer Science, 4(11), pp.1629-1641.

Gupta, P.S. and Gupta, A.S., 1977. Heat and mass transfer on a stretching sheet with suction or blowing. The Canadian Journal of Chemical Engineering, 55(6), pp.744-746.

Hsiao, K.L., 2010. Mixed convection with radiation effect over a nonlinearly stretching sheet. World Academy of Science, Engineering and Technology, 4, pp.338-342.

Hussanan, A., Salleh, M. Z., Khan, I. and Shafie, S., 2016. Analytical solution for suction and injection flow of a viscoplastic Casson fluid past a stretching surface in the presence of viscous dissipation. Neural Computing and Applications. doi:10.1007/s00521-016-2674-0.

Jat, R. N., Chand, G. and Rajotia, D., 2014. MHD heat and mass transfer for viscous flow over nonlinearly stretching sheet in a porous medium. Thermal Energy and Power Engineering, 3(1), pp.191-197.

Kechil, S. A. and Hashim, I., 2009. Flow and diffusion of chemically reactive species over a nonlinearly stretching sheet immersed in a porous medium. Journal of Porous Media, 12(11), pp.1053-1063.

Kumaran, V. and Ramanaiah, G., 1996. A note on the flow over a stretching sheet. Acta Mechanica, 116(1-4), pp.229-233.

Magyari, E. and Keller, B., 1999. Heat and mass transfer in the boundary layers on an exponentially stretching continuous surface. Journal of Physics D: Applied Physics, 32(5), pp.577-585.

Mahdy, A., 2014. Natural convection flow of a non-Newtonian Casson fluid past a vertical stretching plane with mass transfer. World Science Research Journals, 2(6), pp.099-107. 
Makinde, O. D., 2005. Free convection flow with thermal radiation and mass transfer past a moving vertical porous plate. International Communications in Heat and Mass Transfer, 32(10), pp.1411-1419.

Mangathai, P., Reddy, B. R. and Reddy, G. V. R., 2015. MHD boundary layer flow through a porous medium over a stretching sheet with chemical reaction. International Journal of Mathematical Archive, 6(3), pp.1-10.

Mukhopadhyay, S., 2013. Casson fluid flow and heat transfer over a nonlinearly stretching surface. Chinese Physics B, 22(7), p.074701.

Pramanik, S., 2014. Casson fluid flow and heat transfer past an exponentially porous stretching surface in presence of thermal radiation. Ain Shams Engineering Journal, 5(1), pp.205-212.

Prasad, K. V., Vajravelu, K. and Datti, P. S., 2010. The effects of variable fluid properties on the hydro-magnetic flow and heat transfer over a non-linearly stretching sheet. International Journal of Thermal Sciences, 49(3), pp.603-610.

Raju, C. S. K. and Sandeep, N., 2017. Unsteady Casson nano fluid flow over a rotating cone in a rotating frame filled with ferrous nanoparticles: A numerical study. Journal of Magnetism and Magnetic Materials, 421, pp.216-224.
Raptis, A. and Perdikis, C., 2006. Viscous flow over a non-linearly stretching sheet in the presence of a chemical reaction and magnetic field. International Journal of Non-Linear Mechanics, 41(4), pp.527-529.

Reher, E. O., Haroske, D. and Kohler, K., 1969. Chem Technol. Chem Technol, 21(3), pp.137- 143.

Sajid, M. and Hayat, T., 2008. Influence of thermal radiation on the boundary layer flow due to an exponentially stretching sheet. International Communications in Heat and Mass Transfer, 35(3), pp.347-356.

Sakiadis, B., 1961. Boundary-layer behavior on continuous solid surfaces: II The boundary layer on a continuous flat surface. AIChE Journal, 7(2), pp.221-225.

Shehzad, S. A., Hayat, T., Qasim, M. and Asghar, S., 2013. Effects of mass transfer on MHD flow of Casson fluid with chemical reaction and suction. Brazilian Journal of Chemical Engineering, 30(01), pp.187-195.

Singh, S., 2011. Clinical significance of aspirin on blood flow through stenotic blood vessels. Journal of Biomimetics, Biomaterials, and Tissue Engineering, 10, pp.17-24.

Vajravelu, K., 2001. Viscous flow over a nonlinearly stretching sheet. Applied Mathematics and Computation, 124(3), pp.281-288. 